\title{
Studies of the In Vivo Metabolism of
}

\section{Mevalonic Acid in the Normal Rat}

\author{
KJell H. Hellstrom, Marvin D. Siperstein, Lee A. Bricker, and \\ LYNNE J. LUBY \\ From the Department of Internal Medicine, The University of Texas South- \\ western Medical School at Dallas, Dallas, Texas 75235
}

\begin{abstract}
A в S T R A C T Studies were performed to examine synthesis, tissue localization, and metabolism of mevalonic acid in normal rats. Circulating mevalonate was found to have a rapid turnover phase of $5 \mathrm{~min}$ and a slower phase of 40-50 min. Under in vitro conditions the synthesis of mevalonate is carried out most actively by the liver and only to a minor extent by the other tissues studied. The most unexpected finding of this study was that both in vivo and in vitro the kidneys rather than the liver are the primary site of the metabolism of circulating mevalonate. Whereas mevalonate in the liver is rapidly transformed to cholesterol, the major products of mevalonate metabolism in the renal tissues during the same time period are squalene and lanosterol. Exogenous in contrast to circulating mevalonate is metabolized primarily in the intestine.
\end{abstract}

\section{INTRODUCTION}

There is now abundant evidence that mevalonic acid is an essential intermediate in the synthesis of all animal and plant sterols $(1,2)$. In addition, previous studies from this laboratory have established that the reaction responsible for the synthesis of mevalonate represents the primary site of feedback control of cholesterol biosynthesis $(3,4)$, a finding that has been confirmed by a number of subsequent reports (5-7). Finally, this feedback regulation of mevalonate has been shown by both in

Dr. Hellstrom's present address is Department of Medicine, Serafimerlasarettet, 11283 Stockholm, Sweden, and Dr. Bricker's present address is The University of Miami School of Medicine, P. O. Box 875, Biscayne Annex, Miami, Fla.

During these studies, Dr. Hellstrom was supported by U. S. Public Health Service Research Fellowship Award TW 891, and Dr. Bricker was supported by U. S. Public Health Service Training Grant CA 05200. Dr. Siperstein is the recipient of a U. S. Public Health Service Research Career Award HE 1958.

Received for publication 21 December 1971 and in revised form 29 Jamuary 1973. vitro (8-11) and in vivo studies (12) to be regularly lost following malignant change in liver. This lesion, moreover, remains the single known example of the constant loss of a normal feedback system in cancerous tissue (13).

The finding that mevalonic acid plays so key a regulatory function in sterol synthesis serves to emphasize the need for a better knowledge of the metabolic fate of this compound than is currently available. While numerous studies have examined this problem in isolated in vitro systems $(1,2,14-16)$, relatively few studies have been directed at the more physiologic question of the metabolic fate of mevalonic acid in the intact animal.

Gould and Popjak (17) first observed in 1957 that injected DL- $\left[{ }^{14} \mathrm{C}\right]$ mevalonic acid tended to concentrate in the liver of the rat. Subsequently, Goodman, Avigan, and Steinberg (18) confirmed and expanded this observation by demonstrating that in the rat intravenously administered DL- $\left[2-{ }^{14} \mathrm{C}\right]$ mevalonate was rapidly taken up by the liver and there converted into the nonsaponifiable intermediates of cholesterol synthesis. Finally, Garattini, Paoletti, and Paoletti (19) also noted that $2 \mathrm{~h}$ after the administration of $\left[{ }^{14} \mathrm{C}\right]$ mevalonate to rats the label was found primarily in the liver.

It is noteworthy that, while each of these earlier studies indicated that intravenously administered mevalonate is metabolized by the liver, only the study of Garattini et al. (19) attempted to assess the relative importance of the other tissues of the body in the overall metabolism of mevalonate.

It was the purpose of the present study to determine whether organs other than the liver play a role in the metabolism of endogenous mevalonate. The unexpected result of these studies is that the major portion of circulating mevalonate is metabolized not by the liver but by kidneys.

\section{METHODS}

Animals. Unless otherwise indicated the rats employed in this study were $180-200 \mathrm{~g}$ adult females of the Sprague- 
Dawley strain. Both before and during the experiment they were fed $a d l i b$. ground B \& D rat cubes ${ }^{1}$ and water. At the completion of the studies the animals were killed by decapitation, and the organs to be analyzed were immediately excised and prepared as described below.

The labeled mevalonate was dissolved in saline and administered to the animals, as noted, by intravenous or intraperitoneal injection or by a gastric tube. When the mevalonate was given parenterally, the animals were lightly anesthesized with ethyl ether just prior to the study.

Materials. DL- $\left[2-{ }^{14} \mathrm{C}\right]$ mevalonate, specific activity 5.03 $\mathrm{mCi} / \mathrm{mmol}$, DL- $\left[1-{ }^{14} \mathrm{C}\right]$ mevalonate, specific activity of 1.54 $\mathrm{mCi} / \mathrm{mmol}$, and $\mathrm{DL}-\left[5_{-}{ }^{3} \mathrm{H}\right]$ mevalonate with a specific activity of $50 \mathrm{mCi} / \mathrm{mmol}$ were purchased from the Nuclear Chicago Corp. ${ }^{2}$ and were used as their potassium salts. [2${ }^{14} \mathrm{C}$ ] acetate with a specific activity of $2 \mathrm{mCi} / \mathrm{mmol}$ was obtained from New England Nuclear. After treatment with acid both labeled mevalonate preparations were shown to contain at least $95 \%$ of their label in the mevalonolactone peak isolated by gas-liquid chromatography (21).

$\mathrm{L}-\left[1-{ }^{14} \mathrm{C}\right]$ mevalonate was prepared from $\mathrm{DL}-\left[1-{ }^{14} \mathrm{C}\right]$ mevalonate as follows : $20 \mu \mathrm{Ci}(2 \mathrm{mg})$ of $\mathrm{DL}-\left[1-{ }^{14} \mathrm{C}\right]$ mevalonate was dissolved in $3.7 \mathrm{ml}$ of water containing $500 \mu \mathrm{mol}$ of potassium phosphate, $\mathrm{pH} 6.7,50 \mu \mathrm{mol}$ of ATP, and $5 \mathrm{mg}$ of yeast L-mevalonate kinase, prepared as described by the method of Tchen (22) through the protamine sulfate precipitation step. Following incubation for $1 \mathrm{~h}$ at $37^{\circ} \mathrm{C}$ the crude mixture was subjected to thin-layer chromatography on silica gel $\mathrm{G}$ employing butanol: $\mathrm{H}_{2} \mathrm{O}$ : ammonium hydroxide, $60: 30: 10$, as the developing system. The D-mevalonate and unreacted L-mevalonate moved to the front of the thin layer chromatograph in this system. The location of $\mathrm{L}-\left[1-{ }^{14} \mathrm{C}\right]$ mevalonate-PO $\mathrm{R}_{\mathrm{f}}$ of 0.72 was then detected by autoradiography on nonscreen monopak film. The identity of the mevalonate phosphate was confirmed by use of a standard $\mathrm{L}$-mevalonic acid-1 ${ }^{32} \mathrm{PO}_{4}$ synthesized by the Lmevalonate kinase reaction with $\mathrm{ATP}-\gamma-{ }^{32} \mathrm{P}$ and nonradioactive DL-mevalonate.

The band of silica gel containing the $\mathrm{L}-\left[1{ }^{14} \mathrm{C}\right]$ mevalonate$\mathrm{PO}_{4}$ was scraped from the thin-layer chromatograph, and the radioactivity was eluted with $50 \%$ ethanol. The L-[1${ }^{14} \mathrm{C}$ ]mevalonate- $\mathrm{PO}_{4}$ was next incubated with alkaline phosphatase $^{3} 2.4 \mathrm{U}$ for $4 \mathrm{~h}$ at $\mathrm{pH} 7.6$ to remove the phosphate. The free $\mathrm{L}-\left[1-{ }^{14} \mathrm{C}\right]$ mevalonate was then rechromatographed in the same solvent system, and the free mevalonate at the front was recovered by elution of the silica gel with Nanograde methanol. Analysis of the product by gas-liquid chromatography demonstrated that at least $90 \%$ of the recovered ${ }^{14} \mathrm{C}$ was present in mevalonic acid.

Analytical methods. The various tissues to be studied were cut into small pieces and extracted for $2 \mathrm{~h}$ by refluxing repeatedly with chloroform-methanol ( $1: 1$ by volume). A portion of this extract, one-fourth to one-third, was then transferred into a $50 \mathrm{ml}$ screw tube and evaporated to dryness under a stream of nitrogen. $2 \mathrm{ml}$ of $1 \mathrm{~N}$ potassium hydroxide in $35 \%$ ethanol were added per gram of tissue represented in the evaporated extract. The tubes were then flushed with nitrogen, closed with a screw cap and placed in a waterbath $\left(70^{\circ} \mathrm{C}\right)$ for $1 \mathrm{~h}$. The lipids were next separated into the nonsaponifiable fraction, the allyl pyrophosphates and the prenoic acids by the method described by Goodman and Popjak (16). After extraction of

${ }^{1}$ B \& D Mills, Inc., Grapevine, Tex.

${ }^{2}$ Nuclear Chicago Corp., Des Plaines, III.

${ }^{3}$ Calf intestine alkaline phosphatase obtained from Worthington Biochemical Corp., Freehold, N. J. the nonsaponifiable material with petroleum ether, the water phase was acidified to $\mathrm{pH} 1$ and left at room temperature overnight. On the next day the aqueous phase was again made alkaline, and the hydrolized prenoic alcohols were extracted with petroleum ether. The prenoic acids were obtained after a final acidification of the aqueous phase and repeated extractions with petroleum ether. Each petroleum ether fraction was washed twice with a small volume of water, which after backwashing with petroleum ether was added to the original water phase. The petroleum ether extracts were then combined and evaporated under nitrogen. Since the alcohols contained negligible amounts of radioactivity, the acids and alcohols were extracted together in most of the experiments. The nonsaponifiable material was next redissolved in a small volume of chloroform and fractionated by thin-layer chromatography on $20 \times 20 \mathrm{~cm}$ plates coated with silica gel $G$ (plate thickness $250 \mu \mathrm{m}$ ). The chromatograms were developed for $1 \mathrm{~h}$ in heptane-benzene (97:3). Squalene ${ }^{4}$ was identified as a distinct spot at $R_{\mathbf{t}}$ 0.94 , nerolidol $\left(R_{f} \quad 0.72\right.$ ), lanosterol and dihydrolanosterol ( $\left.R_{\mathfrak{f}} 0.63\right)$, farnesol and methostenol $\left(R_{\mathfrak{f}} 0.48-0.54\right)$, geraniol and 27 carbon sterols $\left(R_{f} 0.4\right)$. It was assumed that the 27 carbon sterol band represented cholesterol and no further purification of this compound was carried out. The various bands were visualized by spraying with $0.05 \%$ solution of rhodamine $\mathrm{G}$ in $50 \%$ ethanol and scraped from the plates directly into the scintillation fluid consisting of $0.3 \%$ diphenyloxazole and $0.015 \% p$-bis-phenyloxazolyl-benzene in toluene. Radioactivity was determined with either Packard ${ }^{5}$ or Beckman Liquid Scintillation Counters. ${ }^{6}$

The turnover times of DL- and L-mevalonate were determined as follows: Cannulae of P-10 polyethylene tubing were inserted into the femoral arteries and the tail veins of lightly anesthetized rats. The rats were then placed in restraining cages and allowed to recover for at least $1 \mathrm{~h}$. The labeled mevalonate was then injected through the venous cannulae, and at regular intervals $50 \mu \mathrm{l}$ of blood was collected from the femoral artery and added to $1.0 \mathrm{ml}$ of $0.9 \%$ saline. A $0.6 \mathrm{ml}$ portion of the diluted blood was then directly assayed for ${ }^{14} \mathrm{C}$ using Beckman Bio-Solve $3^{6}$ as the scintillation solution.

Mevalonate synthesis by tissue slices was determined exactly as described in a previous publication (3). The further details of the individual experiments are described under Results.

\section{RESULTS}

\section{Kinetic studies of mevalonate in vivo}

Turnover time of mevalonate in normal rats. The initial experiments of this study were designed to determine the rate at which circulating mevalonate is metabolized in the normal rat. The turnover time of a relatively small dose of mevalonate was estimated by injecting 7.2 $\mu \mathrm{g}(2 \mu \mathrm{Ci})$ of $\mathrm{DL}\left[5-{ }^{3} \mathrm{H}\right]$ mevalonate intravenously into a

4 The methostenol was a gift from Dr. Ivan Franz; the dihydrolanosterol a gift from Dr. Jean D. Wilson. Farnesol and geraniol were obtained from Aldrich Chemical Co., Inc., Milwaukee, Wisc., and lanosterol was purchased from Mann Research Laboratories, New York. The squalene and cholesterol were obtained from Eastman Kodak Co., Rochester, N. Y.

${ }^{5}$ Packard Instrument Co., Inc., Downers Grove, Ill.

${ }^{\circ}$ Beckman Instruments, Inc., Fullerton, Calif. 


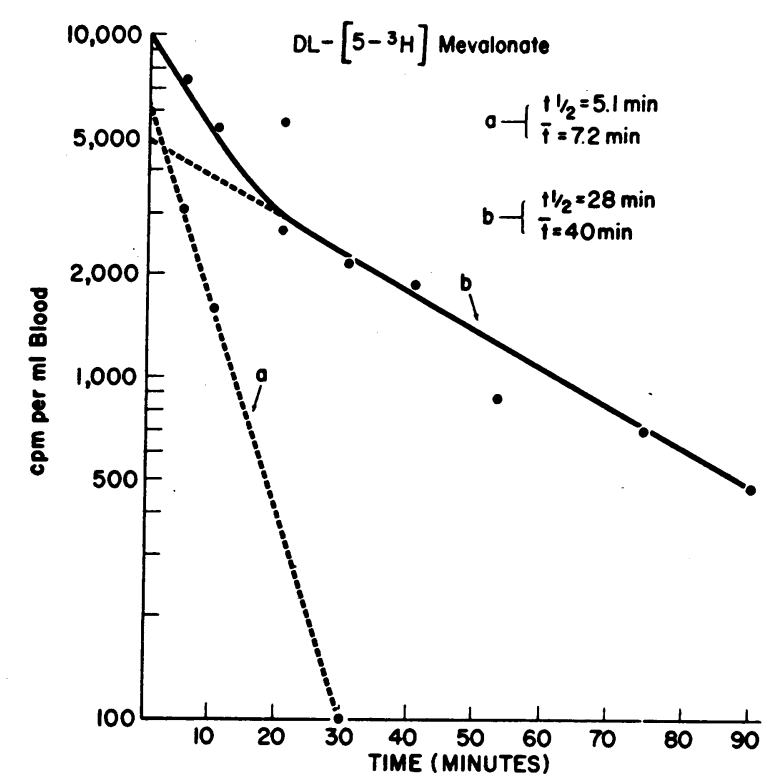

FIGURE 1 Turnover study of $\mathrm{DL}-\left[5-{ }^{3} \mathrm{H}\right]$ mevalonate in a normal rat. The rapid phase of mevalonate turnover was calculated using the method of Gurpide et al. (20).

$200 \mathrm{~g}$ Sprague-Dawley rat. The resulting die-away curves were analyzed according to the two-pool model described by Gurpide, Mann, and Sandberg (20). After an initial rapid decrease in blood radioactivity with a corrected turnover time that can be approximated at 7 min (Fig. 1), a somewhat slower turnover rate with a half-time of $28 \mathrm{~min}$ and a turnover time of $40 \mathrm{~min}$ was observed.

Both because of the possibility of the incorporation of $\left[{ }^{3} \mathrm{H}\right]$ mevalonate into circulating labeled compounds other than mevalonate and because of the complicating problem of the nonphysiologic D-mevalonate influencing the turnover kinetics of administered DL-mevalonate, these turnover studies were repeated using the physiologically active L-isomer of mevalonate, prepared as described under Methods. In addition, in this experiment the mevalonate was labeled in the carboxyl carbon. The metabolism of mevalonate to isopentenyl pyrophosphate results in the loss of its first carbon as ${ }^{14} \mathrm{CO}_{2}$, which in turn is diluted by the large body pool of ${ }^{12} \mathrm{CO}_{2}$ and expired; as a result it would be highly unlikely that significant amounts of ${ }^{14} \mathrm{C}$ present in the blood of the rat injected with $\mathrm{L}-\left[1{ }^{14} \mathrm{C}\right]$ mevalonate would be present in compounds other than mevalonate itself. As shown in Fig. 2, studies utilizing $\mathrm{L}-\left[1-{ }^{14} \mathrm{C}\right]$ mevalonate indicate a rapid turnover phase of $7 \mathrm{~min}$ and a slower component with a turnover time of $52 \mathrm{~min}$, figures that do not differ greatly from the turnover times observed with DL- $\left[5-{ }^{3} \mathrm{H}\right]$ mevalonate.

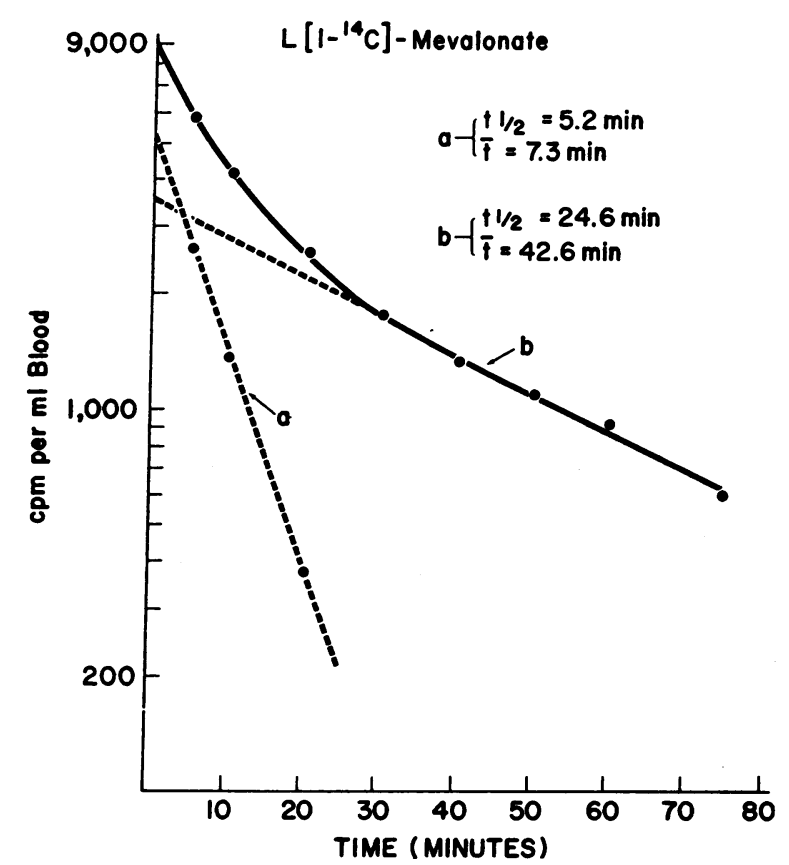

FIGURE 2 Turnover study of $\mathrm{L}-\left[1-{ }^{14} \mathrm{C}\right]$ mevalonate in a normal rat.

\section{Metabolism of endogenous mevalonate}

Tissue distribution of circulating mevalonate. Studies were next carried out to determine where within the body the metabolism of this circulating endogenous pool of mevalonate takes place. The initial experiments were designed to determine the overall tissue distribution and relative affinities of various organs for endogenous mevalonic acid. Accordingly, DL- $\left[2-{ }^{14} \mathrm{C}\right]$ mevalonate (150 $\mu \mathrm{g}$ or $75 \mu \mathrm{g}$ of $\mathrm{L}$-mevalonate) were injected intravenously into rats, the animals were killed $2 \mathrm{~h}$ later, and the total ${ }^{14} \mathrm{C}$ recovered in the various tissues following chloroform and methanol extraction was determined. The results shown in Fig. 3 indicate that, contrary to expectation,

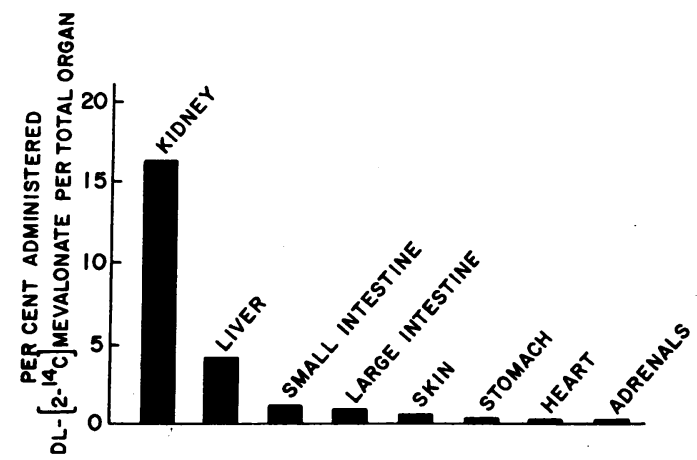

FIgURE 3 Total organ distribution of $\left[{ }^{14} \mathrm{C}\right]$ mevalonate and labeled metabolites $2 \mathrm{~h}$ following intravenous administration of $\mathrm{DL}-\left[2-{ }^{14} \mathrm{C}\right]$ mevalonate.

Mevalonic Acid Metabolism In Vivo 


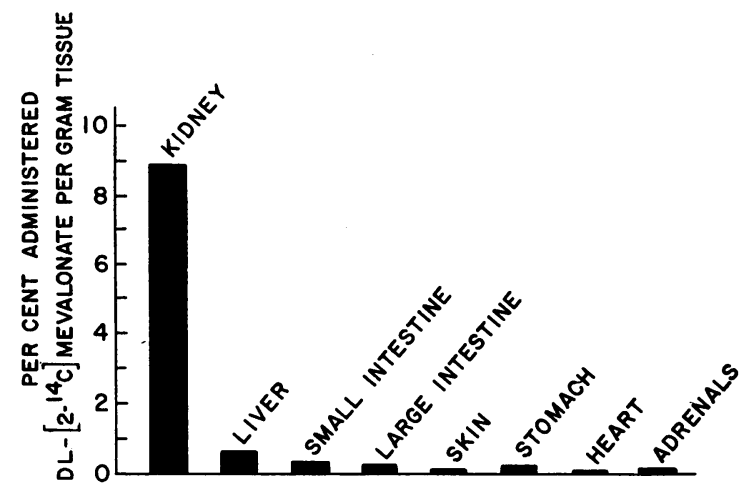

FIGURE 4 Distribution of $\left[{ }^{14} \mathrm{C}\right]$ mevalonate and labeled metabolites per gram of tissue $2 \mathrm{~h}$ following intravenous administration of DL- $\left[2-{ }^{14} \mathrm{C}\right]$ mevalonate.

the kidneys, rather than the liver, represent the major site of uptake of the ${ }^{14} \mathrm{C}$, with four times as much of the label being present in the kidney $(16 \%)$ as was found in the liver $(3.9 \%)$. The entire gastrointestinal tract and its contents accumulated a total of only $2.4 \%$ of the recovered radioactivity with lesser amounts of radioactivity being found in the other tissues examined. It was assumed that the remainder of the mevalonate and its metabolites were either distributed throughout the carcass, which was not examined, or else excreted in the urine.

The ability of the kidneys to accumulate mevalonate and its metabolites is even more strikingly demonstrated when the distribution of the label is calculated on the basis of the weight of the tissues studied. As is shown in Fig. 4, the kidneys per gram of tissue concentrate $\left[{ }^{14} \mathrm{C}\right]$ mevalonate to an extent at least 20 times that observed in the liver.

Tissue localization of mevalonate metabolites at various time intervals. While observations of the previous experiments strongly suggest that the kidneys play an important role in the metabolism of circulating mevalonate, it was conceivable that the localization of ${ }^{14} \mathrm{C}$ in the kidneys represented simply the renal concentration of mevalonate itself, particularly of the unmetabolized D-mevalonate, rather than the actual metabolism of mevalonate by this organ. To resolve this question the ${ }^{14} \mathrm{C}$ present within the kidney, liver, and intestine was fractionated four separate times as described under Methods, into total nonsaponifiable lipids, allyl pyrophosphates, and prenoic acids.

The data in Fig. 5 demonstrate that within $30 \mathrm{~min}$ and at each time interval thereafter a large percentage of the total radioactivity present in the kidney can be accounted for by the metabolites of mevalonate. This conclusion has been confirmed by the observation that only traces of free $\left[{ }^{14} \mathrm{C}\right]$ mevalonate can be recovered in the kidneys by gas chromatography (21) $2 \mathrm{~h}$ after injection of the labeled mevalonate. After $2 \mathrm{~h}$ over $75 \%$ of the remaining $\left[{ }^{14} \mathrm{C}\right]$ mevalonate can be recovered in the urine. Moreover, analysis of the urine by gas-liquid chromatography (21) has demonstrated that within the limits of the method, $100 \%$ of the label in the urine is present as free mevalonic acid. It is likely that, as suggested by Gould and Popjak (17), this radioactivity represents primarily the D-isomer of $\left[{ }^{14} \mathrm{C}\right]$ mevalonate. The relative importance of the kidneys in the metabolism of mevalonate is emphasized by comparison of the total metabolites of mevalonate present in the kidney, with

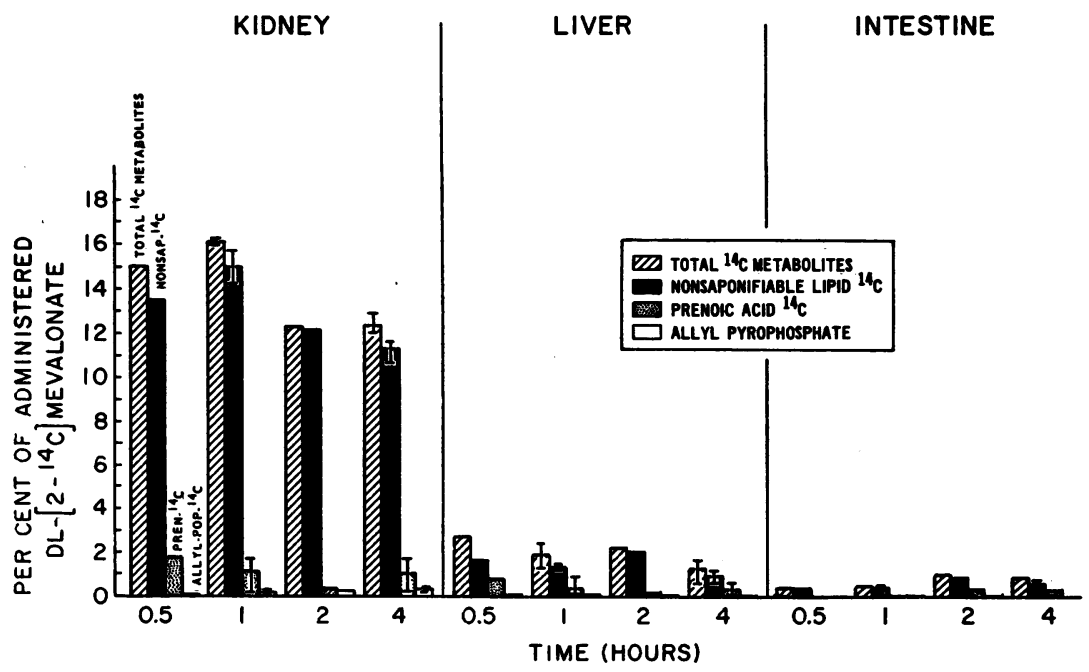

FIGURE 5 Fractionation of mevalonate metabolites following intravenous administration of DL- $\left[2-{ }^{14} \mathrm{C}\right]$ mevalonate. 


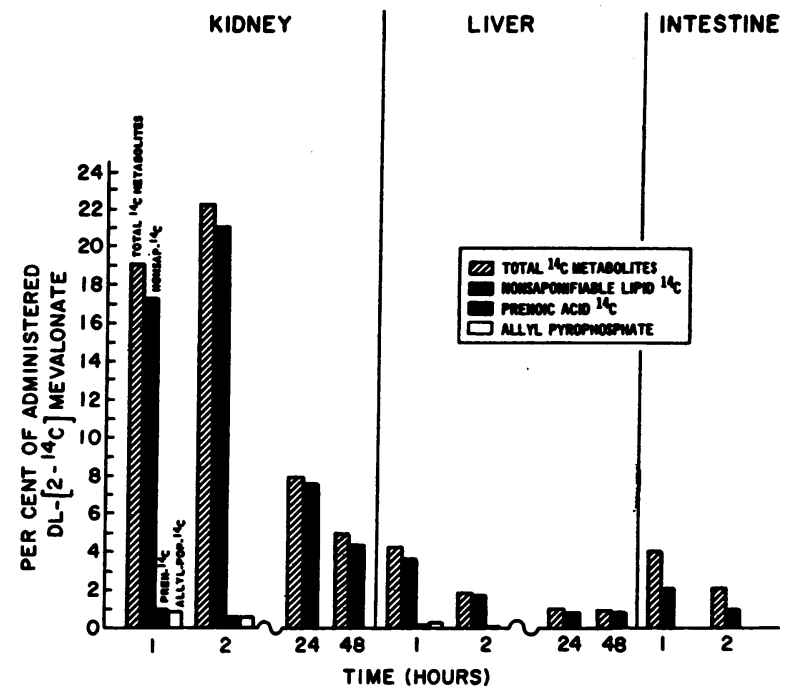

FIGURE 6 Fractionation of mevalonate metabolites following intraperitoneal administration of $\mathrm{DL}-\left[2-{ }^{14} \mathrm{C}\right]$ mevalonate.

those of the liver and intestine (hatched bars, Fig. 5). By 30 min the kidneys were found to contain $15 \%$ of the injected $\left[{ }^{14} \mathrm{C}\right]$ mevalonate as mevalonate metabolites. Since only the $\mathrm{L}$ form of mevalonate is metabolized in the body, actually $30 \%$ of the "metabolizable" mevalonate is present in the kidneys at this time interval. By contrast, the liver, $30 \mathrm{~min}$ after injection of mevalonate, contained only $3 \%$ of the administered ${ }^{14} \mathrm{C}$ as metabolic products of mevalonate, while the intestine accumulated only $0.5 \%$ of the label during this $30 \mathrm{~min}$ period. Moreover, this predominance of the kidneys in mevalonate metabolism persists throughout the 1 st and $2 \mathrm{nd} \mathrm{h}$ after administration of the $\left[{ }^{14} \mathrm{C}\right]$ mevalonate, and in fact the relative concentration of the label in the kidneys is more marked at the 4th $\mathrm{h}$ of the study, at which time over 20 times the amount of ${ }^{14} \mathrm{C}$ is found in the kidneys than is present in the liver.

The data in Fig. 5 also document that the majority, averaging over $90 \%$, of these renal metabolites of mevalonate is recoverable in the nonsaponifiable lipid fraction with only a few percent noted in the acidic or allyl pyrophosphate fractions. This distribution of mevalonate metabolites in the kidney was observed as early as $30 \mathrm{~min}$ after injecting the $\left[{ }^{\mathrm{I}} \mathrm{C}\right]$ mevalonate and persisted throughout the entire $4 \mathrm{~h}$ of observation. A slightly higher percentage of the ${ }^{14} \mathrm{C}$ was noted in the acidic fraction of the liver; however, even in this tissue it is clear that the nonsaponifiable fraction contains the majority of mevalonate metabolites. Finally, in the intestine the predominant mevalonate metabolites were again recovered in the nonsaponifiable fraction.

Subsequent to the above studies it was recognized that, while the amounts of mevalonate employed are much less tabolism, these levels of mevalonate probably far exceed than those used in previous studies of mevalonate methe extracellular mevalonate pool; however, studies employing tracer amounts of DL-mevalonate $(0.5 \mu \mathrm{g}$ or 0.25 $\mu \mathrm{g}$ of L-mevalonate) have given very similar results, i.e. in $2 \mathrm{~h} 12 \%$ of the injected $\mathrm{DL}-\left[2-{ }^{14} \mathrm{C}\right]$ mevalonate was metabolized by kidneys and $3 \%$ in the liver.

As indicated in Fig. 6, when the labeled mevalonate was administered by the intraperitoneal route, an identical tissue distribution of ${ }^{14} \mathrm{C}$ was observed even after 48 h. Moreover, the pattern of mevalonate metabolism, with the predominance of label in the nonsaponifiable fraction of the kidneys, which was observed following the intravenous administration of mevalonate, was confirmed when the labeled mevalonate was given by the intraperitoneal route.

Taken together these studies therefore provide strong evidence not only that the kidney serves as the major tissue of mevalonate uptake but that in addition the kidney may well be the major site of metabolism of circulating endogenous mevalonate.

Subfractionation of nonsaponifiable lipids derived from $\left[2-{ }^{14} \mathrm{C}\right]$ mevalonate. To establish whether the kidneys play a role in sterol metabolism, it was necessary to demonstrate that there is a renal conversion of circulating mevalonate to precursors of cholesterol or to cholesterol itself. Consequently the nonsaponifiable ${ }^{14} \mathrm{C}$ recovered following the intravenous injection of $0.5 \mu \mathrm{g}$ of $\left[2{ }^{14} \mathrm{C}\right]$ mevalonate was subfractionated by thin-layer chromatography, as described under Methods, and the bands corresponding to squalene, lanosterol, and cholesterol were assayed for ${ }^{14} \mathrm{C}$. The results (Fig. 7) indicate a marked qualitative difference between kidney and liver in the metabolism of circulating mevalonate. Within $30 \mathrm{~min}$ $65 \%$ of the $\left[{ }^{14} \mathrm{C}\right]$ mevalonate present in the nonsaponifiable fraction of the kidney has been incorporated into squalene with $21 \%$ in lanosterol and only $10 \%$ in cho-

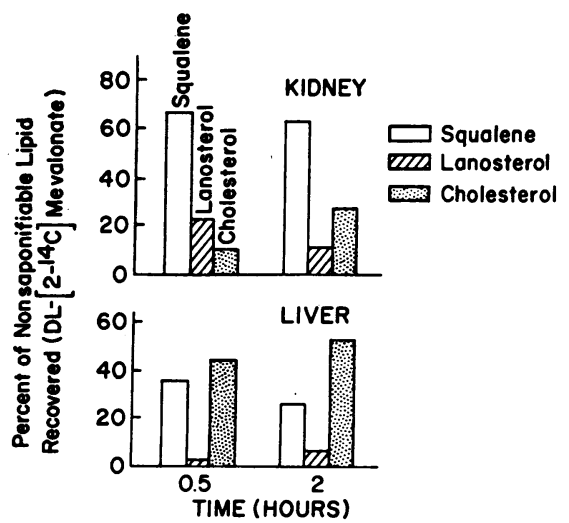

FIgURE 7 Subfractionation of nonsaponifiable metabolites of DL- $\left[2-{ }^{14} \mathrm{C}\right]$ mevalonate following intravenous administration.

Mevalonic Acid Metabolism In Vivo 


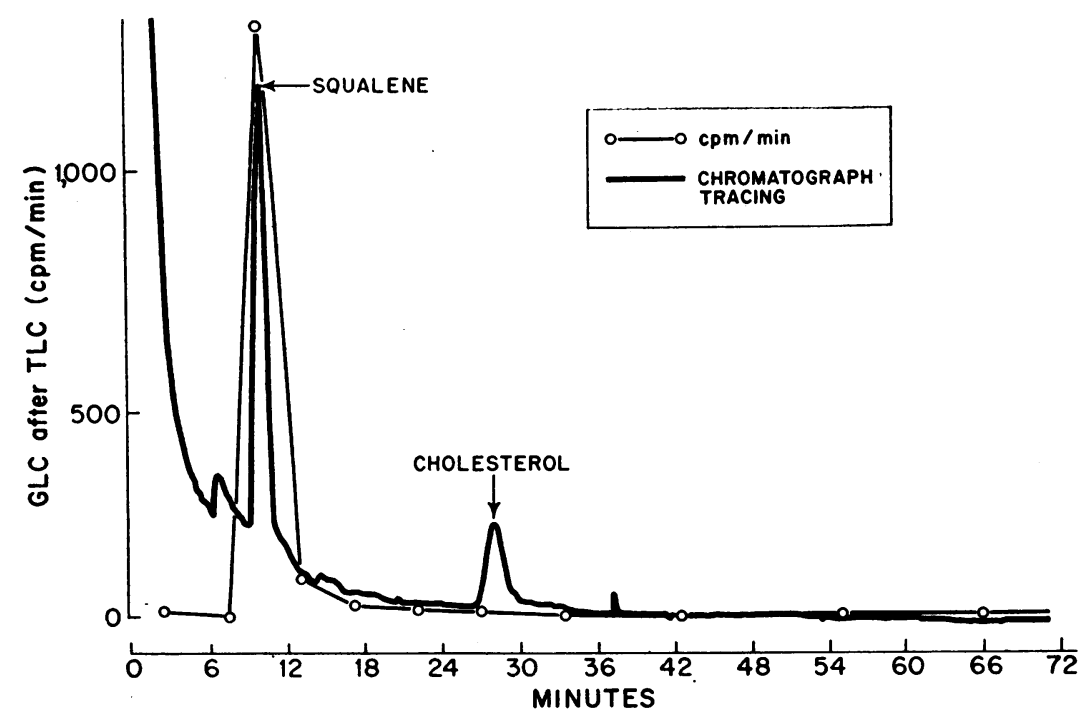

FIGURE 8 Gas-liquid chromatographic identification of squalene as the major metabolite of mevalonate in the kidney.

lesterol. By contrast, even at the earliest interval studied the liver had incorporated the injected mevalonate primarily into cholesterol. This general pattern of mevalonate metabolism was maintained both in the kidneys and liver $2 \mathrm{~h}$ after mevalonate administration.

In view of the importance of the identification of squalene as the major end-product of mevalonate in the kidneys, the squalene band isolated by thin-layer chromatography was further identified by gas-liqid chromatography using OV-17 as the stationary phase, temperature $216^{\circ} \mathrm{C}$, gas flow $100 \mathrm{ml} / \mathrm{min}$. As shown in Fig. 8, squalene under these conditions has an $R_{f}$ of $9 \mathrm{~min}$, and as is apparent at least $95 \%$ of radioactivity characterized as squalene on thin-layer chromatography is in fact found in the squalene peak of the gas-liquid chromato-

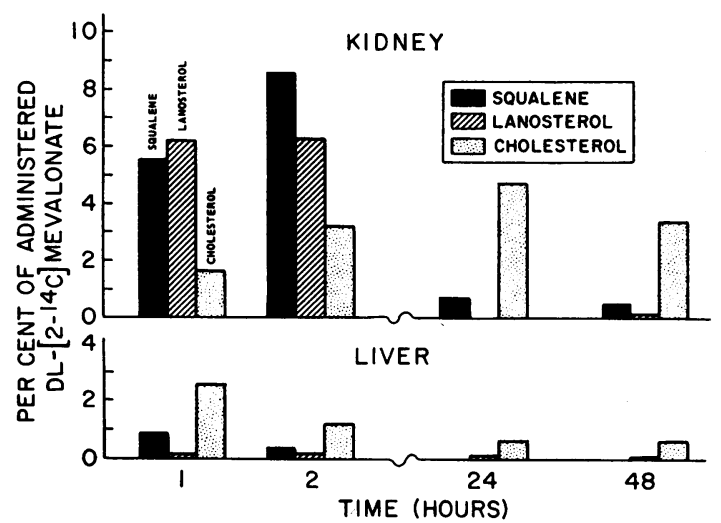

FIGURE 9 Subfractionation of nonsaponifiable lipids in kidney and liver following intraperitoneal administration of DL$\left[2-{ }^{14} \mathrm{C}\right]$ mevalonate. gram. An incidental finding of this gas-liquid chromatographic analysis of squalene is that when analyzed without carrier squalene, the natural concentration of squalene in renal tissue is approximately $14 \mu \mathrm{g}$ per gram of tissue.

Examination of the nonsaponifiable end-products of mevalonate metabolism was carried out at longer intervals, 24 and $48 \mathrm{~h}$, following the administration of the mevalonate by the intraperitoneal route (Fig. 9). At the earlier time intervals, i.e. 1 and $2 \mathrm{~h}$, the mevalonate metabolites in renal tissue conform to those found following the intravenous administration of mevalonate in that the synthesis of squalene and lanosterol by the kidney represents the predominant fate of the mevalonate. At 24 and $48 \mathrm{~h}$, however, ${ }^{14} \mathrm{C}$ in these precursors of cholesterol has decreased markedly, and the preponderance of radioactivity is now found in 27 carbon sterols, presumably cholesterol itself.

The metabolism of substrate amounts of circulating mevalonate. In order to determine whether larger concentrations of mevalonate would be similarly metabolized, the fate of this compound was examined in animals receiving $1 \mathrm{mg}$ of $\mathrm{DL}-\left[2-{ }^{14} \mathrm{C}\right]$ mevalonate intravenously.

As indicated in Fig. 10, the distribution and metabolism of mevalonate under these conditions is very different from that noted with smaller amounts of mevalonic acid, in that at the $2 \mathrm{~h}$ time interval studied, the liver rather than the kidneys represents the major site of mevalonate metabolism. In addition, the metabolism of mevalonate in the liver, as expected, results in a preponderance of the mevalonate metabolites being present in the 27 carbon sterols, presumably cholesterol. The 
finding that the metabolism of these large, probably unphysiologic, doses of mevalonate leads to the liver assuming the major role in mevalonate uptake may serve to reconcile the results of the present studies with those of previous investigators (19) in which the distribution of only relatively large doses of mevalonate was studied.

In vitro metabolism of mevalonate by kidney and liver

Metabolism of DL- $\left[2-{ }^{14} \mathrm{C}\right]$ mevalonate. To rule out the possibility that the mevalonate metabolites found in the kidneys might be derived via the blood stream from the liver, studies were carried out under in vitro conditions in an attempt to determine whether in fact the kidney can, in the absence of liver, actively convert mevalonate into sterols and sterol precursors. $500 \mathrm{mg}$ of either kidney or liver slices were incubated as described under Methods with tracer doses of $\left[2-{ }^{14} \mathrm{C}\right]$ mevalonate for $2 \mathrm{~h}$; the nonsaponifiable lipid fraction was isolated, assayed for ${ }^{14} \mathrm{C}$, and then further subfractionated into its sterol components. The results demonstrate first (upper half of Fig. 11) that kidney slices are capable of significant conversion of mevalonate into nonsaponifiable lipids. In two experiments the kidneys incorporated 17 and $19 \%$ of the added mevalonate to nonsaponifiable sterols while the comparable figures for the liver were 20 and $29 \%$.

As expected the conversion of $\left[{ }^{14} \mathrm{C}\right]$ acetate to nonsaponifiable sterols was significantly less than for mevalonate in each of the two tissues. However, while the liver converted $2 \%$ of the added acetate to nonsaponifiable lipids, the kidney was capable of incorporating only an insignificant $0.03 \%$ of the acetate into this fraction.

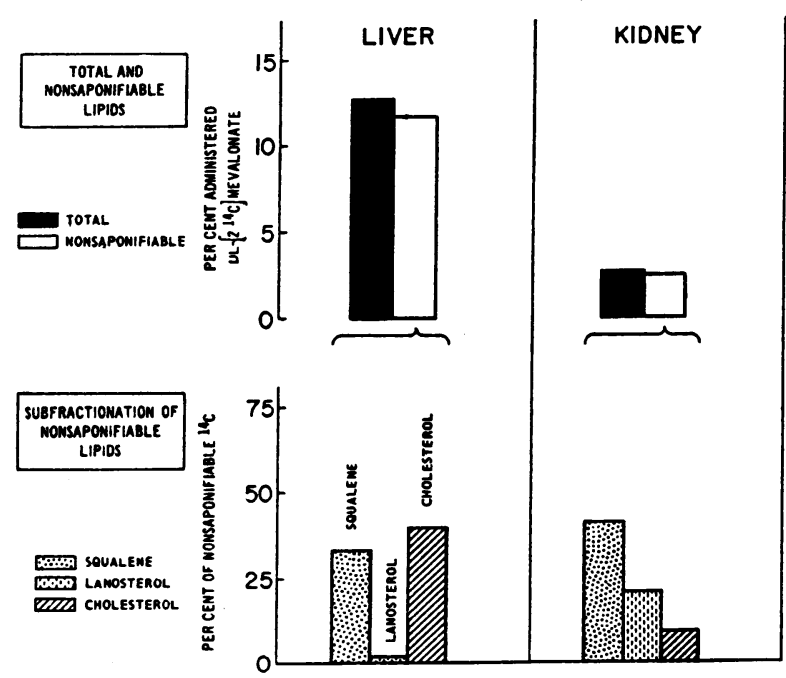

FIgURE 10 Distribution and metabolism of substrate amounts of $\mathrm{DL}-\left[2{ }^{14} \mathrm{C}\right] \mathrm{mevalonate} 2 \mathrm{~h}$ following intravenous administration.

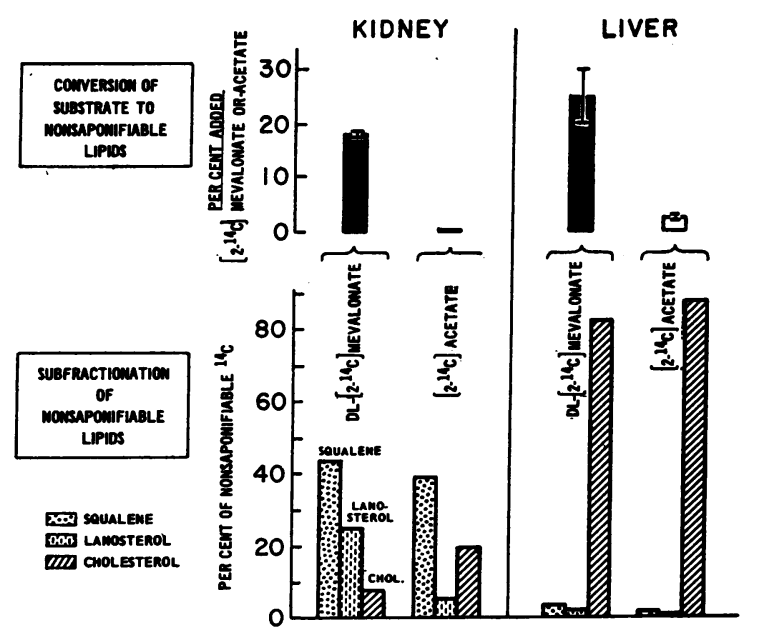

FIGURE 11 Synthesis of nonsaponifiable lipids from DL-[2$\left.{ }^{14} \mathrm{C}\right]$ mevalonate by kidney and liver slices in vitro.

The latter finding would suggest that the kidney has only a limited ability to synthesize mevalonate from acetate, a suggestion that will be confirmed directly in a later experiment (see below). On the other hand, the subsequent steps in sterol synthesis that are responsible for the conversion of mevalonate to nonsaponifiable cholesterol precursors are obviously carried out effectively by renal tissue.

The latter conclusion was supported by the subfractionation of the nonsaponifiable lipid synthesized by the kidney, an analysis that led to results (lower half of Fig. 11) almost identical to those observed in the in vivo experiments. Specifically, the ability of the kidney to convert mevalonate predominately to squalene and lanosterol rather than to cholesterol is again confirmed; and this same pattern is demonstrated with $\left[2-{ }^{14} \mathrm{C}\right]$ acetate as the labeled substrate. As is well known, liver slices are readily capable of incorporating both acetate and mevalonate into cholesterol, and this finding is likewise confirmed by the results of the studies in Fig. 11.

In vitro metabolism of $\mathrm{L}-\left[1-{ }^{11} \mathrm{C}\right]$ mevalonate. An independent means of determining the relative abilities of various tissues to metabolize mevalonate became available with the synthesis of $\mathrm{L}-\left[1-{ }^{14} \mathrm{C}\right]$ mevalonate (see Methods). With the use of this label the conversion of the mevalonate to isoprenoid units could be simply and directly assayed by determining the ability of the kidney and liver to convert $\left[1-{ }^{14} \mathrm{C}\right]$ mevalonate to ${ }^{14} \mathrm{CO}_{2}$. Results of such an experiment are presented in Table I. Again, it is apparent that per gram of tissue the kidney is approximately as capable as is the liver of converting the physiologically active form of mevalonate to isoprene compounds.

Mevalonic Acid Metabolism In Vivo 
TABLE I

Metabolism of $L-\left[1-{ }^{14} \mathrm{C}\right]$ mevalonate to ${ }^{14} \mathrm{CO}_{2}$

\begin{tabular}{lcc}
\hline Organ & $\begin{array}{c}\text { Percent } \\
\text { added }{ }^{4} \mathrm{C}\end{array}$ & $\begin{array}{c}\text { Nanomoles/ } \\
\text { gram } \\
\text { tissue }\end{array}$ \\
\hline Liver & 28.6 & 22.9 \\
Kidney & 25.8 & 20.6 \\
Intestine & 2.7 & 2.2 \\
Spleen & 1.7 & 1.4
\end{tabular}

$200 \mathrm{mg}$ of tissue slices incubated with $16 \mathrm{nmol}$ of $\mathrm{L}-\left[1-{ }^{14} \mathrm{C}\right]-$ mevalonate for $1 \mathrm{~h}$ in Krebs' phosphate buffer $\mathrm{pH} 7.0 .{ }^{14} \mathrm{CO}_{2}$ was collected as previously described (3).

\section{Sites of endogenous mevalonate production}

The observation that plasma mevalonate represents an actively turning over pool, which is metabolized in the kidney, raises the question as to the tissue of origin of this endogenous mevalonate. Slices from a number of tissues were therefore incubated with $\left[1-{ }^{14} \mathrm{C}\right]$ acetate in the presence of an unlabeled pool of mevalonate and the incorporation of the labeled acetate into mevalonate was determined as described in earlier studies $(4,21)$. As shown in Table II, of the tissues studied, liver has by far the greatest ability to synthesize mevalonic acid from acetate; the kidney and testes in fact had only a barely detectable rate of mevalonate synthesis, which averaged at most $1 / 20$ of the liver. The finding that the kidney synthesizes mevalonate at only a minimal rate was of course anticipated by the observation, described in an earlier section of this study, that the conversion of acetate to sterols procedes very slowly in renal tissue.

\section{Metabolism of exogenous mevalonate}

Metabolism of orally administered DL- $\left[2{ }^{14} C\right]$ mevalonate. To study the metabolic fate of exogenous mevalonate, DL- $\left[2-{ }^{14} \mathrm{C}\right]$ mevalonate in a tracer dose was administered by stomach tube to $200 \mathrm{~g}$ Sprague-Dawley
TABLE II

Mevalonate Synthesis in Rat Tissue Slices

\begin{tabular}{llc}
\hline & $\begin{array}{c}{[2-\mu \mathrm{C}] \text { acetate }} \\
\text { converted to } \\
\text { mevalonate/g } \\
\text { tissue/h }\end{array}$ \\
\hline Tissue & 14.0 \\
Liver & 0.6 \\
Kidney & 0.6 \\
Testes & 0.2 \\
Skin & 0 \\
Spleen & 0 \\
Brain & \\
\hline
\end{tabular}

Incubation conditions as described under Methods.

rats, and $2 \mathrm{~h}$ later the animals were killed and the distribution of ${ }^{14} \mathrm{C}$ determined as previously described. The results presented in Fig. 12 demonstrate a rather striking difference in the sites of metabolism of exogenous mevalonate as compared with that of endogenous origin. While, as emphasized above, systemically administered mevalonate is metabolized primarily by the kidneys, the results in the upper half of Fig. 12 demonstrate that after $2 \mathrm{~h}$ the majority of the orally administered $\left[{ }^{14} \mathrm{C}\right]$ mevalonate is present in the intestinal wall and in the liver, with significant but still lesser amounts of ${ }^{14} \mathrm{C}$ metabolites being found in the kidneys.

The label in each of these tissues, including the intestine, is found primarily in the nonsaponifiable lipids indicating that the intestine itself must have metabolized the mevalonate delivered to it from the lumen. Moreover, fractionation of the nonsaponifiable fraction of the mevalonate metabolites, as demonstrated in the lower half of Fig. 12, demonstrates that the $\left[{ }^{14} \mathrm{C}\right]$ mevalonate in the intestine was present primarily as cholesterol. This was also the case with the liver. On the other hand the exogenous mevalonate reaching the kidney is metabolized, as was endogenous mevalonate, primarily to squalene and lanosterol during the time interval studied.
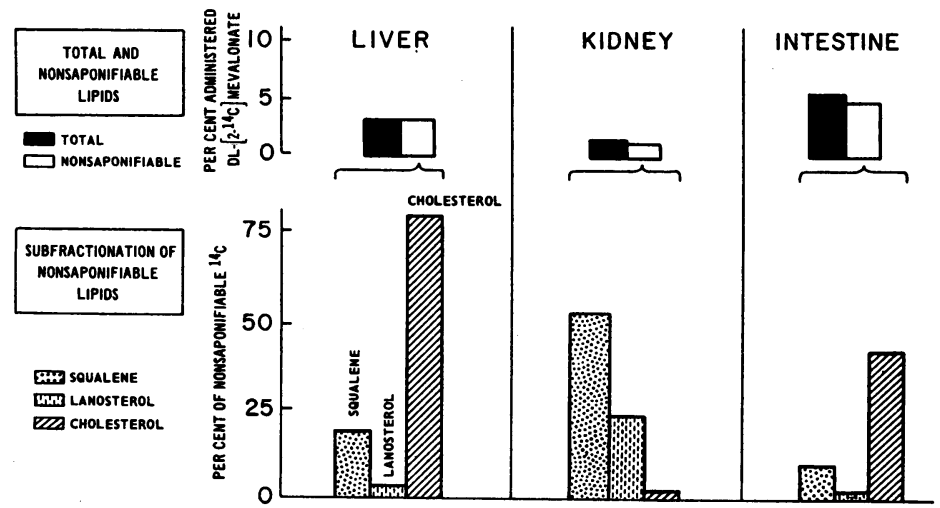

FIGURE 12 Distribution of metabolites of $\left[2-{ }^{14} \mathrm{C}\right]$ mevalonate $2 \mathrm{~h}$ after oral administration. 


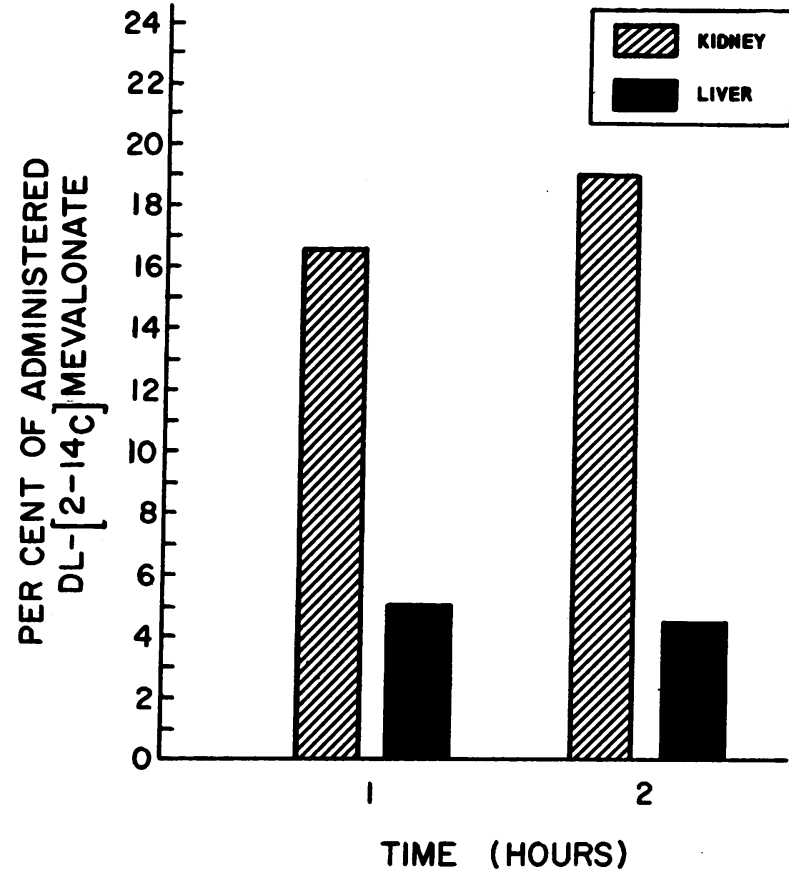

FIGURE 13 Distribution of $\mathrm{DL}-\left[2-{ }^{14} \mathrm{C}\right]$ mevalonate following intraportal injection.

These data suggest that orally administered mevalonate is largely metabolized by the intestine to nonsaponifiable endproducts that would be delivered, primarily via the lymph, to the systemic circulation and from there taken up preferentially by the liver. Alternately, mevalonate might be absorbed by the portal circulation and hence delivered and metabolized by the liver, and only secondarily by the kidney. To examine this question a tracer dose of $\left[2-{ }^{14} \mathrm{C}\right]$ mevalonate was administered through an intestinal venule into the portal circulation, and the distribution of lipid ${ }^{14} \mathrm{C}$ was determined. As shown in Fig. 13 , at both 1 and $2 \mathrm{~h}$ the kidney is the major site of mevalonate concentration; and as was observed following administration of mevalonate by the systemic route, the vast majority of the label present in the kidney has been converted to nonsaponifiable compounds. While the ratio of ${ }^{14} \mathrm{C}$ in the kidney to that in the liver is between 3 and 4:1 rather than the 5 and $7: 1$ ratio observed following the systemic administration of labeled mevalonate, it is still apparent that most of the mevalonate administered into the portal vein must pass through the liver to be delivered to and metabolized primarily by the kidneys. It is very likely therefore that the exogenous mevalonate that is adsorbed as such from the intestine must, like endogenous mevalonate, be chiefly metabolized in the kidneys.

Most exogenous mevalonate, however, is metabolized by the intestine before it can reach the systemic circula- tion. This conclusion was further supported by experiments in which a lymph cannula was inserted into a rat and the percent of administered mevalonate that was absorbed as nonsaponifiable lipid via the lymph determined. The results shown in Table III demonstrate that over $35 \%$ of the total administered DL-mevalonate or over $70 \%$ of the L-mevalonate in the racemic mixture has been converted to nonsaponifiable material, in all probability by the intestine during the $6 \mathrm{~h}$ period of the experiment; moreover, of this nonsaponifiable sterol, over $18 \%$ had been delivered to the lymph during the $6 \mathrm{~h}$ of the experiment. Finally, it is of interest that subfractionation of nonsaponifiable ${ }^{14} \mathrm{C}$ in the lymph reveals that approximately $80 \%$ of this lipid is located in the squalene band on thin-layer chromatography.

It would seem highly likely, therefore, that exogenous mevalonate must be primarily metabolized by the intestine to cholesterol and squalene during the process of absorption. The latter lipid is then delivered to the systemic circulation via the lymph and in all probability is subsequently further metabolized by the liver to cholesterol.

\section{DISCUSSION}

The primary purpose of the present study was to determine the metabolic fate of circulating mevalonic acid in the intact animal.

Clearly, the most unexpected finding to develop from this investigation is that the kidneys represent the major organ responsible for the metabolism of circulating mevalonic acid. Despite the fact that, in contrast to the liver, the kidney has almost no capacity to synthesize mevalonate, $60-70 \%$ of the $\left[{ }^{14} \mathrm{C}\right]$ mevalonate recovered, whether the labeled compound was given intravenously or intraperitoneally, is found in the kidneys while less than $20 \%$ is accounted for in the liver. This ability of the kidney to take up mevalonate was even more clearly illustrated by the fact that per gram of tissue the kidney concentrates $\left[{ }^{14} \mathrm{C}\right]$ mevalonate to an

TABLE III

Conversion of Orally Administered $\left[2{ }^{14} \mathrm{C}\right]$ mevalonate into Nonsaponifiable Lipids in a Rat with a Lymph Fistula for $6 h$

\begin{tabular}{lrccc}
\hline & Liver & Kidney & Intestine & Lymph \\
\hline Nonsaponifiable* & 1.6 & 1.1 & 15.4 & 18.6 \\
Subfraction $\ddagger$ & & & & \\
$\quad$ Squalene & 5.0 & 39.0 & 16.0 & 80.0 \\
Lanosterol & 1.0 & 28.0 & 3.0 & 9.0 \\
Cholesterol & 80.0 & 27.0 & 62.0 & 6.0 \\
\hline
\end{tabular}

* Percent administered $\left[{ }^{14} \mathrm{C}\right]$ mevalonate in nonsaponifiable lipids.

$\ddagger$ Percent of total nonsaponifiable lipids.

Mevalonic Acid Metabolism In Vivo 
extent 10 times that of the liver. More important, almost all of the mevalonate retained by the kidney was found to be rapidly converted into the precursors of cholesterol, specifically squalene and lanosterol, a finding that would indicate that the kidney represents not only a major site of mevalonate uptake but is also the primary organ in which circulating mevalonate is metabolized in the body.

These in vivo studies suggest that while the kidney contains an active mechanism for converting mevalonate to squalene and lanosterol, the further conversion of these intermediates to cholesterol procedes slowly in this tissue. This observation was confirmed in tissue slices in which it could be demonstrated that mevalonate is incorporated into squalene at rates per gram of tissue approximately equal to that of liver; however, the kidney, in contrast to liver, converts squalene and lanosterol to cholesterol to an insignificant extent. The studies of Gold and Olson (23) have shown that kidney slices convert mevalonate to Coenzyme $Q_{\ominus}$ at a rate significantly more rapid than that of heart or liver. The synthesis of Coenzyme $Q$ was not measured in the present study; however, since Gold and Olson observed that the conversion of mevalonate to Coenzyme $Q$ in kidney slices progressed at a rate less than $1 / 10$ that of cholesterol and since the present studies in turn demonstrate that the incorporation of mevalonate into cholesterol amounts to only $1 / 10$ the conversion of mevalonate into nonsaponifiable material, Coenzyme $Q$ synthesis could account for only a very minor fraction, approximately $1 \%$, of the mevalonate metabolized by renal tissue.

The findings of this study obviously raise the question of the role played by the kidneys in the overall metabolism of cholesterol, a question that can only be partially resolved at present. In studies to be published in detail elsewhere), ${ }^{7}$ the concentration of mevalonate in the blood was determined using thin-layer and gas-liquid chromatography and analyzed by mass spectroscopy. In the normal rat, blood mevalonate is present at a level of approximately $5 \mu \mathrm{g} / 100 \mathrm{ml}$. At a blood volume of $9.2 \mathrm{ml}$ in a $200 \mathrm{~g}$ rat (24) the amount of mevalonate in the circulation totals about $0.46 \mu \mathrm{g}$; assuming an equilibrium throughout the extracellular space of $24 \%$ of body weight (25), the mevalonate present in this volume would amount to $2.4 \mu \mathrm{g}$. On the basis of the rapid turnover phase of the $\mathrm{L}-\left[1-{ }^{14} \mathrm{C}\right]$ mevalonate die-away curve of 5 min, which probably represents this rapidly miscible pool, the turnover rate of mevalonate through the extracellular space would be $0.7 \mathrm{mg} /$ day.

\footnotetext{
${ }^{7}$ Bricker, L. A., K. J. Hellstrom, and M. D. Siperstein. A method for measuring tissue levels of mevalonic acid. In preparation.
}

A reasonable estimate of total $24 \mathrm{~h}$ sterol production by a $200 \mathrm{~g}$ rat is approximately $6 \mathrm{mg}$ (26), and if, as is generally assumed, mevalonate is an obligatory intermediate in sterol synthesis it would follow that about 14 $\mathrm{mg}$ of mevalonate would be required to statisfy this daily sterol requirement. If it is assumed that this figure approximates the total mevalonate produced per day, then the above data would indicate that about $5 \%$ of the mevalonate escapes, presumably largely from the liver, into the plasma to comprise the circulating miscible mevalonate pool examined in the present study. It is possible that the kidney utilizes this circulating mevalonate pool to synthesis small amounts of cholesterol for structural purposes. On the other hand, both the in vivo and in vitro results demonstrate that the kidney converts squalene and lanosterol to cholesterol at a slow rate, and the evidence from the present study demonstrates that the kidney does not excrete detectable amounts of these mevalonate metabolites into the urine. It seems not improbable, therefore, that the kidney may release a portion of these cholesterol precursors into the circulation to be carried to the liver, there finally to be converted to cholesterol. Goodman's finding that significant quantities of squalene are present in the blood of both man and rat lends some support to the likelihood of such an active circulating pool of squalene in these species (27).

It should be emphasized that, while the above conclusions are based largely on the tissue distribution of injected DL-mevalonate, i.e. the mixture of the metabolically inactive $\mathrm{D}$-isomer and the active $\mathrm{L}$-isomer, it is likely that the findings are physiologically sound. Examination of the tissue distribution of the metabolites of mevalonate, which could be derived only from the L-isomer, confirms the fact that the kidney is the major site of mevalonate metabolism; and secondly, the turnover studies using the physiologically active L-isomer of mevalonate confirmed the turnover data obtained initially with the racemic mevalonate.

The metabolism of exogenous mevalonate differs from that of the endogenously derived compound in that, when ingested in the diet, this sterol precuror is no longer metabolized primarily by the kidneys. Ingested mevalonate is largely converted in the intestinal wall to nonsaponifiable lipids, which are then absorbed via the lymph into the systemic circulation. It is likely therefore that the intestine rather than the kidneys plays the more important role in the primary metabolism of the small quantities of mevalonate that may be consumed in the diet.

Finally, the conclusions of this study are restricted at present only to the rat, and it remains to be determined whether the importance of the kidney in mevalonate metabolism, which has been demonstrated in this study, can be applied to other species as well. 


\section{ACKNOWLEDGMENTS}

We wish to express our appreciation for invaluable assistance in the study to Dr. Millie Wiley, Marianne McKissock, Susie Abright, Judith Hedderman, Judy Johns, Violet Fagan, Ann Gyde, Alice Clark, and Carol Jenkins.

This work was supported by U. S. Public Health Service Grant CA 08501, and by Damon Runyon Memorial Fund for Cancer Research Grant 747.

\section{REFERENCES}

1. Tavormina, P. A. 1956. The utilization of $\beta$-hydroxy- $\beta$ methyl- $\delta$-valerolactone in cholesterol biosynthesis. J. $\mathrm{Am}$. Chem. Soc. 78 : 4498.

2. Lynen, F., H. Eggerer, U. Henning, and I. Kessel. 1958. Farnesyl-pyrophosphat und 3-methyl- $\Delta^{3}$-butenyl-1-pyrophosphat, die biologischen vorstufen des squalens. Angew. Chem. 70: 738 .

3. Siperstein, M. D., and M. J. Guest. 1960. Studies on the site of the feedback control of cholesterol synthesis. J. Clin. Invest. 39: 642.

4. Siperstein, M. D., and V. M. Fagan. Feedback control of mevalonate synthesis by dietary cholesterol. J. Biol. Chem. 241: 602 .

5. Linn, T. 1967. The effect of cholesterol feeding and fasting upon $\beta$-hydroxy- $\beta$-methylglutaryl CoA reductase. J. Biol. Chem. 242: 990.

6. Shapiro, D. J., and V. W. Rodwell. 1969. Diurnal variation and cholesterol regulation of hepatic HMG-CoA reductase activity. Biochem. Biophys. Res. Commun. 37: 867.

7. White, L. W., and H. Rudney. 1970. Regulation of 3hydroxy-3-methylglutarate and mevalonate biosynthesis by rat liver homogenates. Effects of fasting, cholesterol feeding, and triton administration. Biochemistry. 9: 2725.

8. Siperstein, M. D., and V. M. Fagan. 1964. Deletion of the cholesterol-negative feedback system in liver tumors. Cancer Res. 24: 1108.

9. Siperstein, M. D., V. M. Fagan, and H. P. Morris. 1966. Further studies on the deletion of the cholesterol feedback system in hepatomas. Cancer Res. 26: 7.

10. Siperstein, M. D. 1965. Comparison of the feedback control of cholesterol metabolism in liver and hepatomas. In Developmental and Metabolic Control Mechanisms and Neoplasia. The Williams \& Wilkins Company, Baltimore, Md. 427.

11. Siperstein, M. D., A. M. Gyde, and H. P. Morris. 1971. Loss of feedback control of $\beta$-hydroxymethylglutaryl coenzyme A reductase in hepatomas. Proc. Natl. Acad. Sci. U.S. A. $68: 315$.

12. Bricker, L. A., H. P. Morris, and M. D. Siperstein. 1972. Loss of the cholesterol feedback system in the intact hepatoma-bearing rat. J. Clin. Invest. 51: 206.
13. Pitot, H. C. 1966. Some biochemical aspects of malignancy. Annu. Rev. Biochem. $35: 335$.

14. Amdur, B. H., H. Rilling, and K. Bloch. 1957. The enzymatic conversion of mevalonic acid to squalene. $J$. Am. Chem. Soc. 79: 2646.

15. Popjak, G., L. Gosselin, I. Youhotsky Gore, and R. G. Gould. 1958. Studies on the biosynthesis of cholesterol. 6. Coenzyme requirements of liver enzymes for synthesis of squalene and of sterol from DL-3-hydroxy-3-methyl[2- $\left.{ }^{14} \mathrm{C}\right]$ pentano-5-lactone. Biochem. J. 69: 238

16. Goodman, D. S., and G. Popjak. 1960. Studies on the biosynthesis of cholesterol. XII. Synthesis of allyl pyrophosphates from mevalonate and their conversion into squalene with liver enzymes. J. Lipid Res. 1: 286.

17. Gould, R. G., and G. Popjak. 1957. Biosynthesis of cholesterol in vivo and in vitro from DL- $\beta$-hydroxy- $\beta$ methyl- $\delta-\left[2-{ }^{14} \mathrm{C}\right]$-valerolactone. Biochem. J. 66: 721.

18. Goodman, D. S., J. Avigan, and D. Steinberg. 1963. Studies of cholesterol biosynthesis. V. The time course and pathway of the later stages of cholesterol biosynthesis in the livers of intact rats. J. Biol. Chem. 238: 1287.

19. Garattini, S., P. Paoletti, and R. Paoletti. 1959. Lipid biosynthesis in vivo from acetate-1-C $\mathrm{C}^{\mathbf{1 4}}$ and $2-\mathrm{C}^{\mathbf{1 4}}$ and mevalonic-2-C ${ }^{14}$ acid. Arch. Biochim. Biophys. 84: 253.

20. Gurpide, E., J. Mann, and E. Sandberg. 1964. Determination of kinetic parameters in a two-pool system by administration of one or more tracers. Biochemistry. 3: 1250.

21. Siperstein, M. D., V. M. Fagan, and J. M. Dietschy. 1966. A gas-liquid chromatographic procedure for the measurement of mevalonic acid synthesis. J. Biol. Chem. $241: 597$.

22. Tchen, T. T. 1959. Mevalonic kinase: purification and properties. J. Biol. Chem. 233: 1100.

23. Gold, P. H., and R. E. Olson. 1966. Studies on Coenzyme Q. J. Biol. Chem. 241: 3507.

24. Berlin, N. I., R. L. Huff, D. C. Van Dyke, and T. G. Hennessy. 1949. Blood volume of adult rat, as determined by Fe59 and P32 labeled red cells. Proc. Soc. Exp. Biol. Med. 71: 176.

25. Barratt, T. M., and M. Walser. 1969. Extracellular fluid in individual tissues and in whole animals: the distribution of radiosulfate and radiobromide. J. Clin. Invest. 48: 56.

26. Wilson, J. D. 1964. The quantification of cholesterol excretion and degradation in the isotopic steady state in the rat: the influence of dietary cholesterol. J. Lipid Res. 5 : 409.

27. Goodman, D. S. 1964. Squalene in human and rat blood plasma. J. Clin. Invest. 43:1480. 